\title{
Conductive Lithographic Films
}

\author{
B.J. Ramsey, P.S.A. Evans ${ }_{\left(M_{M B E R}\right)} \&$ D. Harrison \\ Department of Design \\ Brunel University \\ Egham, United Kindom, TW20 0JZ
}

\begin{abstract}
This paper reports progress in the development of a novel fabrication technique for printing circuit board designs directly onto suitable substrates. Circuit tracks can be formed on organic or synthetic substrates by depositing films of a metalloaded ink via a standard lithographic printing process. The application of this work is in substitutes for conventional (copper-clad resin/laminate) circuit boards where, for low complexity circuits, directly printed substrates offer cost advantages and environmental benefits. The paper is a resume of work and results, including; ink formulation, environmental test, circuit modelling and life cycle analysis. Conductive Lithographic Films have now been successfully demonstrated in a telephone handset developed in conjunction with Nortel, microprocessor and microwave stripline applications. Whilst developed primarily as a low cost, low environmental impact alternative to subtractive $P C B$ manufacture, other potential advantages such as flexibility and environmental robustness are apparent.
\end{abstract}

Indexing Terms:- Circuit Boards, Film Circuits, Conductive Inks, Lithography, Microwave Striplines.

\section{Introduction:-}

"One of the most important environmental accomplishments in PWB development would be the establishment of a fully additive printed wiring board (manufacture) process... On average, the waste stream constitutes $92 \%$ - and the final product just $8 \%$ of the total weight of the materials used... Approximately $80 \%$ of the waste produced is hazardous" These extracts taken from the 1996 Electronics Industry Environmental Roadmap summarize the well known fundamental problems with existing, subtractive practices.

Conductive Lithographic Films are an emerging additive fabrication technique for a wide range of electronic circuits and systems. The process employs standard lithographic printing technology to fabricate conductive film patterns on a range of flexible substrates using a purpose-developed conductive ink. Conductive Lithographic Film circuits can be printed rapidly, using fewer processes and a fraction of the materials currently used, and are consequently cheaper and cleaner to produce. The films are also robust, withstanding a range of standard environmental test regimes.

In order to use the offset lithographic process for circuit fabrication, a comprehensive knowledge is needed of all aspects of lithographic printing. Printing success depends on the ink, the substrate, the print plate, the fountain solution and the printing machine. The lithographic printing process offers excellent dimensional control and registration of substrate patterns coupled with high speed production.

\subsection{Conventional Circuit Boards:-}

Interest in circuit substrate fabrication by printing processes has arisen in response to the following factors:

- The cost of manufacturing traditional etched resin/laminate circuit boards. This arises from the large number of discrete manufacturing operations. (e.g.,. Photo-resist processes, etching, through-hole plating, drilling, cropping, silk screening, tin-plating, lacquering and inspection.) PCB manufacture remains a complex and highly specialized process, conducted in isolation from the mainstream of electronic equipment assembly.

- Hazardous waste products from the wet chemical plating, cleaning and etching phases of production, which require specialized treatment.

- Disposal of redundant populated circuit boards. Toxic residues from soldering operations and small quantities of numerous substances present hazards, rendering recycling operations costly and problematic.

It is considered that the disadvantages associated with existing circuit board fabrication techniques can 
be circumvented if the etching phase of the production process can be eliminated and resin/laminate substrate substituted by materials presenting fewer environmental hazards in disposal. Etching operations can be avoided by specifying an additive process to form the conductive track patterns, and by adapting that process to less hazardous substrate materials.

It was recognized that certain printing processes possess desirable characteristics that could be adapted to form the basis of a novel circuit fabrication technology, whilst permitting greater flexibility in the choice of substrate material. The characteristics of the lithographic process were considered particularly suited to circuit fabrication.

\subsection{The Lithographic Printing Process:-}

Lithography relies on the action of two wetting functions on the surface of a smooth and un-embossed printing plate. The plate chemistry repels water where the printed image is dark, allowing an oil-based ink to adhere. A water film repels the ink in light regions of the image. Contact with an ink and a moistening roller allows the printing plate to attract both water and ink as required, and to form the image to be printed. The image is not printed directly onto the substrate material (e.g.,. paper), but is instead transferred to an intermediate or "blanket cylinder" that has a yielding surface. The blanket cylinder then presses the ink film onto the surface of the substrate, which is now supported on a separate impression cylinder. The printed substrates rely on evaporation and/or oxidation of the ink film for the image to become fixed.

Standard lithographic printing machines have the following characteristics:

- High Speed (Typically 3000 - 10000 Impressions/hour).

- Good Dimensional Control \& Excellent Registration of Images.

- Low Cost per Sheet (Low Ink Volume/Substrate).

- Widespread Availability of Necessary Production Facilities.

\section{Circuit Printing:-}

Having identified lithographic printing as a suitable printing process, two components of the circuit fabrication strategy required development, namely:

1. An electrically conductive lithographically printable ink.

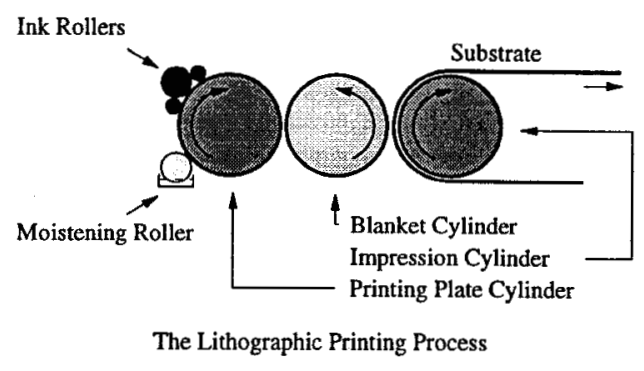

Figure 1: The Basic Lithographic Printing Process.

2. A compatible substrate material on which to print circuit tracks.

Ink: Analysis of ink rheology and conductivity during progressive print trials has resulted in the identification of conductive ink formulations with suitable mechanical and electrical properties. Detailed analysis of results have allowed evaluation of laydown weight, film thickness ( 5 micrometers) and sheet resistivity. The relationship of sheet resistivity to volume fraction of conductor has been established allowing evaluation of the effect of the organic phase on sheet resistivity, independent of laydown weight.

Ink layers deposited by standard printing machines have a thickness of the order of 3 microns. When compared with 50 micron thick conductors $\left(50 \times 10^{-6} \mathrm{~m}\right)$ resulting from screen printing processes, and of the 0.07 to $0.1 \mathrm{~mm}$ of copper laminated onto conventional laminate circuit boards, it was apparent that the developed ink must exhibit high electrical conductivity, whilst conforming to the various mechanical and chemical constraints imposed by the printing process. Double-sided circuits can be printed easily using double sided print machines or by further passes through single-sided machines.

Printed Film Substrates: The type of substrate used must conform to several basic parameters if a printed circuit is to have appropriate electrical characteristics and service life. These include environmental stability, dimensional stability, suitable surface roughness, appropriate hydrophobic and hydrophilic attraction, fire retardency, and suitable dielectric and resistive properties. Synthetic and coated papers have yielded the best results, the former having superior environmental and electrical stability. Enhancements to properties of moisture absorption, fire retardency and chemical attack can be achieved through conformal coatings, impregnations or additional printed layers. The ink formulation has been engineered to be compatible with a wide range of organic and synthetic 
substrates.

Through-hole interconnection is an essential facet of any circuit board fabrication process. There are two methods under currently under investigation, firstly, a process utilizing the printing operation to force ink under pressure through holes in the substrate to create an electrical contact between films printed on alternate sides of the substrate. This is a complex process as the quantity of ink available to form the interconnect is limited by the small volumes transferred by the print process.

An alternative approach is to screen print conductive adhesive into the through-holes as the circuit board is screen printed with conductive adhesive during the board/component assembly process. This is the equivalent stage to screen printing the solder paste in traditional PCB manufacture. This method is currently yielding higher reliability as more conductive adhesive can be applied to each interconnect.

\section{Film Analysis:-}

Circuit Model: In order to develop a circuit model of the films suitable for circuit simulation purposes, a series of tests, including analysis from D.C. to microwave frequencies has been undertaken.

The printed films possess a raw sheet resistivity of $\approx 0.3 \Omega$ per square. A curing process involving the heating of the films to $\approx 80^{\circ} \mathrm{C}$ for a duration of two hours will decrease the sheet resistivity to between 40 and 50 percent of the raw resistivity value, resulting in a cured sheet resistivity of $\approx 0.15 \Omega$ per square.

The electrical characteristics of the deposited films and substrate have been investigated and tested. The constructs behave as a typical metallic conductor and can be described using any standard conductor model. Exact values of resistivity, capacitance and inductance depend upon the particular ink formulation and the substrate printed on.

Microwave Frequencies: The results of tests conducted on strip line resonator circuits to solve the compound dielectric properties of the substrate exhibit resonances similar to copper, though a linear decrease of $1 \mathrm{~dB}$ at $45 \mathrm{MHz}$ to $3 \mathrm{~dB}$ at $20 \mathrm{GHz}$ is apparent. It has also been demonstrated that coplanar waveguide structures can be deformed in three dimensions without a change in characteristics.

Printing Resolution Limits: Current resolution limits of the process lie between $0.1 \mathrm{~mm}$ and $0.01 \mathrm{~mm}$ (100 and 10 microns). The printed films show a track and gap width of $0.1 \mathrm{~mm}(\approx 0.004$ inches $)$ that provides reliable conductivity. Track widths of $0.01 \mathrm{~mm}$ result in a visible although nonconducting deposit. The current limit to resolution is the variation in registration that occurs with over printing, the resolution of the printing plate, and the mechanical characteristics of the ink. Of the machines trialed to date overprint registration has been the dominant factor, causing alignment errors of up to $\approx 30$ microns.

Environmental Test: Environmental testing is currently in progress through collaboration with Nortel. Conductive Lithographic Films (CLFs) have proved more resistant to industrial atmospheres (particularly sulphurous gases) and humidity/temperature cycling than expected.

In order to asses the suitability of the films for typical product lifecycles a series of environmental tests have been conducted on unprotected, bare samples.

1. IEC-68-2-60:pt.2:Ke1995. Flowing Mixed Gas Corrosion (Test Method 1)

2. ISE-62-2-67:pt.2:Cy:1996. Damp Heat, Steady State, Accelerated Test. $85^{\circ} \mathrm{C}-85 \%$ Relative Humidity.

3. IEC-68-2-3:Ca:1969. Damp Heat, Steady State, $40^{\circ} \mathrm{C}-93 \%$ Relative Humidity.

Results show no catastrophic failure of either the film or the substrate. In all cases the resistivities were lower than in the raw state, due to the curing effect. These tests were conducted in an unbiased condition, further tests of biased samples are in progress. Preliminary thermal shock testing of basic board and component assemblies have demonstrated the assembled circuits to be robust and are being used to determine suitable adhesives. Further trials of greater severity and statistically significant sample sizes are planned.

Environmental Impact Analysis: This evaluation is drawn from "Preliminary Assessment of Environmental Performance of Conductive Lithographic Films" available from the authors, which discusses the problems and pitfalls encountered in environmental assessment. As a result of a lack of available data and tools the assessment is simplified to a comparison of hazardous waste created by conventional PCB manufacture and offset litho print shops, and an evaluation of the reduction in processes needed for manufacture.

Waste stream comparison: These figures are based upon three case studies of PCB manufacturers and print shops, as such they give ballpark figures for the two processes. All the studies are of US firms to ensure classifications of hazardous materials are reasonably consistent between studies. To produce $1 \mathrm{~m}^{2}$ of circuit board from bought in copper clad board incurred a hazardous waste disposal cost of $\$ 0.63$. $\$ 0.02$ is a result of the Seal Brite process (the green lacquer layer) applied to provide environmental protection. 


\begin{tabular}{|c|c|c|}
\hline Procens: & Etchiod Circult Board Manufacture & Lhthographicelly printed Circuit Manut. \\
\hline \multirow{2}{*}{$\begin{array}{l}\text { Baso matorlal } \\
\text { Wantufacture }\end{array}$} & Lamination of copper to substrate & Ink Manufiacture \\
\hline & Application of corrosion prevention layer (oft. Zn plate) & NA \\
\hline PCB Manufacture & Board preparation, removal of protective layer & NA \\
\hline \multirow[t]{3}{*}{ Through Holes } & Orilling/punching out of through holes and wob & Drilling/Punching out of through \\
\hline & Desmearing of through holes (wet chemical process) & NA \\
\hline & Through hole plating (various wot chemical processes) & $\mathrm{NA}$ \\
\hline \multirow[t]{3}{*}{ Clicuit Tracks } & Resist deposition (sereened mask of photo exposure) & Print Circuit Pattem \\
\hline & Etch (wet chemical process) & NA \\
\hline & Resist strip (wet chemical process) & NA \\
\hline \multirow[t]{3}{*}{ Protection } & Corrosion protection (wet chemical process) & NA \\
\hline & Seal-brlte soldermask (screen printed) & Sealbrite soldermask (screen printed) \\
\hline & Wave soider to th exposed pads & NA \\
\hline \multirow[t]{3}{*}{ Board Assombly } & Screen print soldar paste & Screen print conductive adhesive \\
\hline & Place components & Place components \\
\hline & Wave solder to tix components & Cure adhesive ( $80^{\circ} \mathrm{C}$ cure) \\
\hline
\end{tabular}

Figure 2: Comparison of manufacturing processes.

To produce $1 \mathrm{~m}^{2}$ of lithographically printed material incurred a $\$ 0.007$ hazardous waste disposal cost. In addition to this, environmental protection using the Seal Brite process would add 2 cents to the cost of hazardous waste disposal. This represents a two orders of magnitude improvement over the etching phase and a single order of magnitude improvement over the whole process.

Reduction of processes: To simplify the analysis of the two processes it is assumed that both run using FR4 or similar substrate, this is a plausible assumption as the lithographic process is flexible enough to print on to a wide range of substrates including standard FR4 type materials. Figure 2 compares the two processes, the PCB manufacture represents a general double-sided board process as illustrated in the 1996 Electronics Industry Environmental Roadmap. The third column demonstrates how lithographically deposited films can replace many of the chemical and water intensive plating and cleaning phases. The remainder of the manufacturing phases can be transferred essentially unaltered to process conductive lithographically deposited films. The table illustrates a minimum risk scenario for the implementation of the process. Higher risk/cost scenarios which would further reduce the environmental impact of manufacture include moves to different substrate materials, and the lithographic printing of the Seal Brite type environmental protection.

\section{Applications:-}

The potential application for this technology is broad, so far the areas of focus have been low specification circuit board replacement, sensors and deformable microwave structures, though it is envisaged that possible applications include light weight circuits and antenna. Several demonstration circuits have been constructed on organic and synthetic substrate materials. All are fully functional and demonstrate the suitability of the process to low cost, low specification applications as a mechanism for reducing the environmental impact during production and end of life disposal.

Each circuit represents an example of the potential for lithographic film circuit technology in different areas.

1. Electronic Telephone Hand Set(Analogue-Digital Systems).

2. Thermometer (Microprocessor Systems).

3. Microwave Stripline Circuits (RF Applications).

These demonstrators have proved successful, required only minimal thickening of some of the circuit board tracks, and have utilized the same value components as the original products. Implementation of through-hole interconnects, double sided boards and surface mount component (SMT) technologies show the potential of the process.

\section{Conclusions:-}

In conclusion, Conductive Lithographic Films offer a lower cost, lower environmental impact alternative to thick film and copper printed circuit boards.

The lithographic printing technique offers a number of advantages over conventional approaches to PCB manufacture.

1. Lower cost

2. High volume process (3000 to 10000 sheets/hour).

3. Circuit track resolution comparable to the best of other processes (currently limited by the type of print machine used).

4. Greater choice of mechanical properties of the substrate (lighter weight, flexibility).

5. Reduced environmental impact (less energy, reduction in material use, easier disposal).

However, these characteristics have to be offset against reduced performance in other areas, including:

1. Sheet resistivities at the high end of thick film applications. $(\approx 0.15 \Omega$ per square) may limit size and component density of high complexity circuits. Reduction of resistivity is one of the key areas currently being researched. 
2. Solder incompatibilities, printed films cannot yet be soldered. However, it is anticipated that solder will gradually be replaced by lower temperature, less harmful alternatives and the demand for solderability will decrease. The demonstrator circuits and assemblies are completely lead free, except for many existing components which have solder tinned contacts to enable them to be soldered to traditional etched boards.

Conductive Lithographic films were originally conceived as a fabrication technique for low-cost lowcomplexity circuits and systems. However, work has demonstrated a wider application than anticipated, including useful applications in low-cost microwave stripline circuits.

\section{Acknowledgements:-}

The Group acknowleges our Industrial Collaborators Gwent Electronic Materials and Nortel Technology (Harlow) who have provided guidance and technical facilities. This research is funded inpart by the EPSRC Electronic Product Design \& Manufacture initiative, and the Clean Technology Unit.

\section{References}

[1] S. Pendersen, C. Willson G. Pitts \& W. Stotesbury Document Number: MCC-ECESM-001-96. 1996 Microelectronics and Computer Technology Corporation. "1996 Electronics Industry Environmental Roadmap"

[2] D. Harrison, E. Billett \& J. Billingsley. Int.l Conference on Clean Electronic Products and Technology. 9th - 11th October 1995, Edinburgh, UK. "Novel Circuit Fabrication Techniques for Reduced Environmental Impact"

[3] P.R. Shepherd., P.S.A. Evans., B.J. Ramsey \& D.J. Harrison. Automated R.F. and Microwave Measurement Society Conference. 31st October 1st November 1996. Limpley Stoke, Bath, UK. "Assessment of Conductive Lithographic Films for Microwave Applications."

[4] S. Leppavuori, J. Vaananen, M. Lahti, J. Remes \& A Uusimaki.

Microelectronics and Material Physics Laboratories, University of Oulu, FIN-90570 Oulo (Finland) Sensors and Actuators A 41 - 42 (1994) 593 - 596 "A Novel Thick Film Technique, Gravure Offset Printing, for the Realization of Fine-line Sensor Structures."

[5] B.J. Ramsey \& P. J. Simpson.

Cleaner Electronics Research, Dept. Design, Brunel University, United Kingdom Jan. 1997. Unpublished "Preliminary Assessment of Environmental Performance of Conductive Lithographic Films."

[6] Gradel, T.E., Allenby, B.R., \& Comrie,P.R. Environmental Science and Technology, p. 134, vol. 29, number 3 .

"Matrix Approaches to Abridged Life Cycle Analysis."

[7] P. Sozeck

IEEE Trans. on Components, Hybrids, and Manufacturing Technology, Vol. 12, No. 2, June 1989 "Two Novel Additive Processes to Manufacture Circuit Boards: Direct Laser Writing and Direct Electrostatic Transfer and Deposition."

[8] R.M. Scarisbrick

J. Phys D: Appl. Phys., Vol. 6, 1973.

Central Electricity Research Laboratories, Leatherhead, Surrey, UK

"Electrically Conducting Mixtures" 